\title{
Correction to: Thoracic and cardiovascular surgery in Japan during 1999
}

\section{Annual report by the Japanese Association for Thoracic Surgery}

Committee of Science $^{1} \cdot$ Keishu Yasuda ${ }^{2} \cdot$ Hiroyoshi Ayabe ${ }^{3} \cdot$ Hiroko Ide $^{4} \cdot$ Soichiro Kitamura $^{5}$

Published online: 6 July 2018

(c) The Japanese Association for Thoracic Surgery 2018

\section{Correction to:}

General Thoracic and Cardiovascular Surgery 1999 https://doi.org/10.1007/BF03400171

The original publication of the article cited above included incorrect values in the following part: in Table 1 of section (C) Esophageal Surgery.

The corrected version is shown in this correction.

The original article can be found online at https://doi.org/10.1007/ BF03400171.

Committee of Science

survey-adm@umin.net

1 Tokyo, Japan

2 Department of Cardiovascular Surgery, Hokkaido University School of Medicine, Sapporo, Japan

3 First Department of Surgery, Nagasaki University School of Medicine, Nagasaki, Japan

4 Department of Surgery, Institute of Gastroenterolgy, Tokyo Women's Medical University, Tokyo, Japan

5 Department of Cardiovascular Surgery, National Cardiovascular Center, Suita, Japan 
Table 1 Benign esophageal diseases

\begin{tabular}{|c|c|c|c|c|c|c|}
\hline & & \multicolumn{4}{|c|}{ Operation (+) } & \multirow[t]{3}{*}{ Operation (-) } \\
\hline & & \multirow[t]{2}{*}{ Total cases } & \multirow[t]{2}{*}{ Endosc. surg. } & \multicolumn{2}{|l|}{30 day death } & \\
\hline & & & & Total cases & Endosc. surg. & \\
\hline 1 & Achalasia & 181 & 60 & 0 & 0 & 40 \\
\hline \multirow[t]{4}{*}{2} & Benign tumor & 123 & 40 & 0 & 0 & 44 \\
\hline & (1) Leiomyoma & 91 & 32 & 0 & 0 & 32 \\
\hline & (2) Cyst & 10 & 3 & 0 & 0 & 8 \\
\hline & (3) Others & 22 & 5 & 0 & 0 & 4 \\
\hline 3 & Diverticulum & 35 & 6 & 1 & 0 & 71 \\
\hline 4 & Haital hernia & 278 & 68 & 2 & 0 & 602 \\
\hline 5 & Spontaneous rupture of the esophagus & 67 & 11 & 4 & 1 & 6 \\
\hline 6 & Esophageal perforation & 39 & 8 & 0 & 0 & 4 \\
\hline 7 & Esophago-tracheal fistula & 32 & 5 & 0 & 0 & 16 \\
\hline 8 & Congenital esophageal atresia & 67 & 1 & 0 & 0 & 3 \\
\hline 9 & Congenital esophageal stenosis & 20 & 4 & 0 & 0 & 2 \\
\hline 10 & Corrosive stricture of the esophagus & 26 & 3 & 0 & 0 & 14 \\
\hline 11 & Esophagitis, Esophageal ulcer & 114 & 25 & 0 & 0 & 686 \\
\hline \multirow[t]{3}{*}{12} & Esophageal varices & 498 & 110 & 1 & 0 & 936 \\
\hline & (1) Laparotomy & 82 & 8 & 1 & 0 & \\
\hline & (2) Sclerotherapy & & & & & 534 \\
\hline \multirow[t]{2}{*}{13} & Others & 19 & 5 & 0 & 0 & 19 \\
\hline & Total & 1499 & $(346)$ & 8 & (1) & 2443 \\
\hline
\end{tabular}

